TRANSACTIONS OF THE

AMERICAN MATHEMATICAL SOCIETY

Volume 352, Number 5, Pages 2389-2405

S 0002-9947(00)02232-7

Article electronically published on February 14, 2000

\title{
ON THE STABLE MODULE CATEGORY OF A SELF-INJECTIVE ALGEBRA
}

\author{
KARIN ERDMANN AND OTTO KERNER
}

\begin{abstract}
Let $\Lambda$ be a finite-dimensional self-injective algebra. We study the dimensions of spaces of stable homomorphisms between indecomposable $\Lambda$ modules which belong to Auslander-Reiten components of the form $\mathbf{Z} A_{\infty}$ or $\mathbf{Z} A_{\infty} /\left\langle\tau^{k}\right\rangle$. The results are applied to representations of finite groups over fields of prime characteristic, especially blocks of wild representation type.
\end{abstract}

We are interested in homological properties of modules for self-injective algebras. An important invariant of a finite-dimensional algebra is its stable Auslander-Reiten quiver; and during the past years, it has played a crucial role for classification problems of self-injective algebras of finite or tame representation type.

We call a stable component quasi-serial, if it is of the form $\mathbf{Z} A_{\infty}$ or $\mathbf{Z} A_{\infty} /\left\langle\tau^{k}\right\rangle$. If $A$ is any tame algebra, then most of its Auslander-Reiten components are homogeneous tubes, by [CB], hence are quasi-serial. Moreover, it seems that for self-injective algebras of wild type most components of the stable Auslander-Reiten quiver are quasi-serial. For a block of a group algebra which is of wild representation type, all components are of this form (see [E2]). Therefore, it is important to understand homological properties of quasi-serial components.

If $\Lambda$ is a finite-dimensional self-injective algebra and $X, Y$ are $\Lambda$-modules, then we denote the stable homomorphisms from $X$ to $Y$ by $\operatorname{Hom}(X, Y)$. Following the terminology in R1], if $M$ is a module in a quasi-serial stable component $\mathcal{C}$, then the quasi-length of $M$ is the number of the row to which $M$ belongs. The module $M$ is quasi-simple if it has quasi-length one, that is, if it lies at the end of the component.

The first two chapters contain basic facts on dimensions of spaces of stable homomorphisms; this is intended to provide tools which may be of more general use.

In Chapter 3 we prove the following general results. Suppose $f$ is an equivalence of the stable module category of $\Lambda$, consider stable homomorphisms $\underline{\operatorname{Hom}}(M, f M)$ for modules $M$ in a quasi-serial component $\mathcal{C}$. We prove that the dimension of $\underline{\operatorname{Hom}}(M, f M)$ is weakly increasing as a function of the quasi-length of $M$, except possibly when $\tau \Omega^{-1} \cong f \neq \tau$ on $\mathcal{C}$ (see 3.3). Moreover, we study the cases when $f=\tau^{s}$ and $f=\Omega^{s}$ in more detail.

We show that if $\mathcal{C}$ is a tube, then the dimensions of $\underline{\operatorname{Hom}}\left(M, \tau^{s} M\right)$ for $M \in \mathcal{C}$ are unbounded (3.5). In general, if $\mathcal{C}$ is a component of the form $\mathbf{Z} A_{\infty}$ which is not fixed by $\Omega$ and the dimensions of $\underline{\operatorname{Hom}}\left(M, \tau^{s} M\right)$ are bounded, then $\underline{\operatorname{Hom}}\left(X, \tau^{-m} X\right)=0$

Received by the editors June 4, 1996 and, in revised form, October 2, 1997.

2000 Mathematics Subject Classification. Primary 18G25; Secondary 16G70, 20C20.

Key words and phrases. Stable category of finite-dimensional self-injective algebras, quasiserial Auslander-Reiten components, blocks of wild type, endo-trivial modules of $p$-group algebras. 
for $m$ large where $X \in \mathcal{C}$ is quasi-simple (see 3.6). Following $[\mathrm{P}]$, an indecomposable module $M$ with $\underline{\operatorname{End}}(M) \cong K$ is called a stable brick. As a consequence we obtain that if $M$ is a stable brick in a quasi-serial component $\mathcal{C}$ as above, then all modules in $\mathcal{C}$ of quasi-length less or equal to the quasi-length of $M$ are also stable bricks.

These results are valid for group algebras. Most important is the case when $\Lambda=K G$ where $G$ is a finite $p$-group and $K$ is a field of characteristic $p$; and for these we obtain stronger results. Assume $\mathcal{C}$ is a quasi-serial component of such an algebra $\Lambda$. We show that then the dimension of $\underline{\operatorname{Hom}}(M, f M)$ is actually strictly increasing as a function of the quasi-length, if $f$ is a stable equivalence fixing $\mathcal{C}$, except possibly when $\Omega \cong f \neq \Omega^{2}$ on $\mathcal{C}$ (4.2); this uses Carlson's theory of rank varieties of modules. It follows that a stable brick in a quasi-serial component must be quasi-simple. Moreover, using properties of the Ext-algebra we show that $\psi$ is at least unbounded in the general case of a wild block of a group algebra. We note that similar properties hold for restricted enveloping algebras.

In [D], Dade introduced endo-trivial modules; these occur in various contexts in the modular representation theory of finite groups. Endo-trivial modules for $p$-group algebras are stable bricks. Hence we have as a consequence that any endotrivial module of a $p$-group algebra of wild representation type is quasi-simple.

Suppose $\Lambda$ is an arbitrary group algebra and $\mathcal{C}$ is a quasi-serial stable AuslanderReiten component of $\Lambda$. We show that then the dimensions of $\underline{\operatorname{Hom}}\left(M, \tau^{s} M\right)$ for $M \in \mathcal{C}$ are always unbounded (4.7).

A motivation for this work was the observation that there are analogies for representations of hereditary algebras and the stable category of group algebras. If $A$ is a wild hereditary algebra, then all Auslander-Reiten components except for two are of the form $\mathbf{Z} A_{\infty}$; see R1. On the other hand, all stable Auslander-Reiten components for a block of a group algebra of wild type are quasi-serial; see [E2]. In [B], [K1] properties of $\underline{\operatorname{Hom}}\left(M, \tau^{s} N\right)$ for regular modules of wild hereditary algebras were obtained which turned out to be of great importance; this suggested a study of $\underline{\operatorname{Hom}}\left(M, \tau^{s} N\right)$ for self-injective algebras. Moreover, some of our results on stable bricks for self-injective algebras are analogues of results in $\mathrm{K} 2$. The methods used here are, however, quite different as those for hereditary algebras.

We assume that the field $K$ is algebraically closed. All algebras have finite dimension over $K$ and are self-injective. Modules are finite-dimensional left modules, and we write homomorphisms to the right. For general properties of the AuslanderReiten quiver and of stable categories for self-injective algebras we refer to [ARS, [B].

\section{ACKNOWLEDGEMENT}

This work was supported by an EPSRC Visiting Fellowship for the second author; this was held at Oxford and we are grateful to the Mathematical Institute at Oxford for their hospitality.

\section{Self-injeCtive ALgebras}

1.1. In this section we will give an outline of general homological properties of the stable category of a self-injective algebra and relate these to the Auslander-Reiten quiver. Recall that the stable category $\underline{\bmod } \Lambda$ has objects such as the $\Lambda$-modules, and if $X, Y$ are $\Lambda$-modules, then the space of morphisms from $X$ to $Y$ in $\underline{\bmod } \Lambda$ is 
defined to be

$$
\underline{\operatorname{Hom}}(X, Y):=\operatorname{Hom}(X, Y) / \mathcal{P}(X, Y)
$$

where $\mathcal{P}(X, Y)$ is the subspace of $\operatorname{Hom}(X, Y)$ of maps factoring through projectives. Since $\Lambda$ is self-injective, we have $\mathcal{P}(X, Y)=\mathcal{I}(X, Y)$, the space of maps factoring through injectives and hence $\underline{\operatorname{Hom}}(X, Y)=\overline{\operatorname{Hom}}(X, Y)(=\operatorname{Hom}(X, Y) / \mathcal{I}(X, Y))$. For $f \in \operatorname{Hom}(X, Y)$ we denote by $f$ its image in $\underline{\operatorname{Hom}}(X, Y)$.

We will very often write $(X, Y)$ for $\operatorname{Hom}_{\Lambda}(X, Y)$, and we write $(X,-)$ and $(-, Y)$ for the corresponding functors.

1.2. Assume $\Lambda$ is a self-injective algebra over $K$. If $M$ is a module. then $\Omega M$ is the kernel of a minimal projective cover for $M$, and $\Omega^{-1} M$ is the cokernel of an injective hull. Then $\Omega$ induces an equivalence of the stable category of $\Lambda$, and so does the Auslander-Reiten translate $\tau$. In particular, $\Omega$ induces a graph isomorphism of the stable Auslander-Reiten quiver of $\Lambda$.

1.3. We shall use the following functorial isomorphisms:

(1) If $X, Y$ are indecomposable, then

$$
D \operatorname{Ext}^{1}(X, Y) \cong \underline{\operatorname{Hom}}\left(\tau^{-1} Y, X\right) \cong \overline{\operatorname{Hom}}(Y, \tau X)
$$

where $D=\operatorname{Hom}(-, K)$ denotes the usual duality. This is the Auslander-Reiten formula. We shall use the fact that $\operatorname{Ext}^{1}(X, Y)$ and $\underline{\operatorname{Hom}}(Y, \tau X)$ and $\underline{\operatorname{Hom}}\left(\tau^{-1} Y, X\right)$ have the same dimension, for $\Lambda$ self-injective; and for simplicity we only use isomorphisms over $K$ and omit $D$. In particular, we have isomorphism over $K$

$$
\underline{\operatorname{End}}(X) \cong \operatorname{Ext}^{1}(X, \tau X) \cong \operatorname{Ext}^{1}\left(\tau^{-} X, X\right) .
$$

(2) Further we will use that

$$
\underline{\operatorname{Hom}}(A, B) \cong \operatorname{Ext}^{1}\left(\Omega^{-1} A, B\right) \cong \operatorname{Ext}^{1}(A, \Omega B),
$$

is natural in $A$ and in $B$.

(3) Moreover, $\operatorname{Ext}^{n}(M, N) \cong \operatorname{Ext}^{n-s}\left(\Omega^{s} M, N\right) \cong \operatorname{Ext}^{n-s}\left(M, \Omega^{-s} N\right)$.

1.4 Lemma. Assume $0 \rightarrow A \stackrel{\epsilon}{\rightarrow} B \stackrel{\pi}{\rightarrow} C \rightarrow 0$ is an exact sequence of $\Lambda$-modules, and suppose $W$ is any $\Lambda$-module. Then the induced sequence

$$
\underline{\operatorname{Hom}}(W, A) \stackrel{\bar{\epsilon}}{\rightarrow} \underline{\operatorname{Hom}}(W, B) \stackrel{\bar{\pi}}{\rightarrow} \underline{\operatorname{Hom}}(W, C)
$$

is exact. Moreover,

(1) $\bar{\epsilon}$ is $1-1$ if and only if $\left(\Omega^{-1} W, \pi\right)$ is onto.

(2) $\bar{\pi}$ is onto if and only if $(W, \pi)$ is onto.

Proof. Apply $\operatorname{Hom}\left(\Omega^{-1} W,-\right)$ to the given exact sequence; since $\underline{\operatorname{Hom}}(W,-)$ is naturally isomorphic to $\operatorname{Ext}^{1}\left(\Omega^{-1} W,-\right)$ we get the first statement from the long exact sequence of homology, and also part (1) follows.

For the second part, we have $\bar{\pi}$ is onto if and only if $\operatorname{Ext}^{2}\left(\Omega^{-1} W, \epsilon\right)$ is 1-1. But $\operatorname{Ext}^{2}\left(\Omega^{-1} W,-\right)$ is naturally isomorphic to $\operatorname{Ext}^{1}(W,-)$; and $\operatorname{Ext}^{1}(W, \epsilon)$ is $1-1$ if and only if $(W, \pi)$ is onto. 
1.4.1. For convenience, we state the dual of 1.4.

There is an exact sequence

$$
\underline{\operatorname{Hom}}(C, W) \stackrel{\bar{\pi}}{\rightarrow} \underline{\operatorname{Hom}}(B, W) \stackrel{\bar{\epsilon}}{\rightarrow} \underline{\operatorname{Hom}}(A, W) .
$$

(1) The map $\bar{\pi}$ is 1-1 if and only if $(\epsilon, \Omega W)$ is onto.

(2) The map $\bar{\epsilon}$ is onto if and only if $(\epsilon, W)$ is onto.

1.4.2 Lemma. Let $0 \rightarrow X \stackrel{f}{\rightarrow} Y \stackrel{g}{\rightarrow} Z \rightarrow 0$ be a short exact sequence.

(a) If $\alpha: Y \rightarrow M$ is a homomorphism with $\underline{\text { fa }}=0$, then there exists $\beta: Z \rightarrow M$ with $\underline{\alpha}=g \beta$.

(b) If $\overline{\alpha:} M \rightarrow Y$ is a homomorphism with $\underline{\alpha g}=0$, then there exists $\beta: M \rightarrow X$ with $\underline{\alpha}=\underline{\beta f}$.

Proof. (a) From Lemma 1.4 we get an exact sequence

$$
\underline{\operatorname{Hom}}(Z, M) \stackrel{\tilde{g}}{\rightarrow} \underline{\operatorname{Hom}}(Y, M) \stackrel{\tilde{f}}{\rightarrow} \underline{\operatorname{Hom}}(X, M) .
$$

By assumption $\underline{\alpha}$ is in the kernel of $\tilde{f}$, hence in the image of $\tilde{g}$, which is the claim. Part (b) is dual.

1.5. Suppose $W$ is a $\Lambda$-module, and denote by $d_{W}$ the function

$$
d_{W}=\operatorname{dim} \underline{\operatorname{Hom}}(W,-) .
$$

It follows from (1.4) that for a short exact sequence $0 \rightarrow A \rightarrow B \rightarrow C \rightarrow 0$ that $d_{W}(B) \leq d_{W}(A)+d_{W}(C)$ always holds.

1.5.1. Then $d_{W}$ is additive on an Auslander-Reiten sequence

$$
0 \rightarrow \tau X \stackrel{\epsilon}{\rightarrow} E \stackrel{\pi}{\rightarrow} X \rightarrow 0
$$

if and only if $X$ is not a summand of $W$ or $\Omega^{-1} W$; this was proved in ES. It also follows from 1.4; since by the hypotheses on $W$ and by the almost split property, both $\left(\Omega^{-1} W, \pi\right)$ and $(W, \pi)$ are onto.

There is a dual of this result; which follows from 1.4.1. Namely, let $d_{W}^{\prime}:=$ $\operatorname{dim} \underline{\operatorname{Hom}}(-, W)$, then $d_{W}^{\prime}$ is additive on Auslander-Reiten sequences

$$
0 \rightarrow Y \rightarrow F \rightarrow \tau^{-} Y \rightarrow 0
$$

for which $Y$ is not a summand of $W$ or $\Omega W$.

We have for indecomposable modules $W, X$ that $d_{W}(X)=d_{\tau W}(\tau X)$ and $d_{W}(X)$ $=d_{\Omega W}(\Omega X)$.

1.5.2. Suppose $W$ is arbitrary and

$$
0 \rightarrow \tau X \stackrel{\epsilon}{\rightarrow} E \stackrel{\pi}{\rightarrow} X \rightarrow 0
$$

is an Auslander-Reiten sequence; this induces always an exact sequence

$$
\underline{\operatorname{Hom}}(W, \tau X) \rightarrow \underline{\operatorname{Hom}}(W, E) \rightarrow \underline{\operatorname{rad}}(W, X) \rightarrow 0 .
$$

This follows from 1.5.1; the image of $\bar{\pi}$ in 1.5.1 is $\underline{\operatorname{rad}}(W, X)$. 


\section{STABLY QUASI-SERIAL COMPONENTS}

2.1. We call a stable component quasi-serial if it is of the form $\mathbf{Z} A_{\infty}$ or $\mathbf{Z} A_{\infty} /\left\langle\tau^{k}\right\rangle$, in analogy to the terminology in [R1]. Moreover, an Auslander- Reiten component is said to be stably quasi-serial if its stable part is quasi-serial.

Let $\mathcal{C}$ be a stable component which is quasi-serial. A module $X$ in $\mathcal{C}$ is called quasi-simple if the middle term of the AR-sequence of $X$ is indecomposable (or indecomposable modulo projectives). That is, $X$ lies at the end of $\mathcal{C}$.

Now suppose that $X$ is quasi-simple. In $\mathcal{C}$ there is a unique infinite sectional path

$$
\cdots \rightarrow[r] X \rightarrow[r-1] X \rightarrow \cdots \rightarrow[2] X \rightarrow[1] X=X .
$$

Then any indecomposable in the component which is not projective has the form $\tau^{i}[r] X$, for some $i \in \mathbf{Z}$. The quasi-length of a module $M$ in $\mathcal{C}$ is the number of the row containing $M$, so $[r] X$ has quasi-length $r$. We write $q l(M)=r$. The quasi-top of $[r] X$ is $X$.

Dually, the module $X(r)$ is defined via the unique infinite sectional path

$$
X=X(1) \rightarrow X(2) \rightarrow \cdots \rightarrow X(r) \rightarrow X(r+1) \rightarrow \cdots .
$$

Then $X(r)$ has quasi-length $r$; and we call $X$ the quasi-socle of $X(r)$. Let $X$ be the quasi-socle of $X(r)$, then the quasi-top of $X(r)$ is $Y$ where $Y \cong \tau^{-r+1} X$ and moreover, $X(r) \cong[r] Y$.

We use the same terminology for the non-projective indecomposable modules in a stably quasi-serial component.

If $\mathcal{C}$ is is quasi-serial, then so is $\Omega \mathcal{C}$; moreover, the quasi-length is invariant under $\Omega$. In the special case of symmetric algebras, if $\mathcal{C} \cong \mathbf{Z} A_{\infty}$, then $\Omega \mathcal{C} \neq \mathcal{C}$; otherwise, one would have $\Omega \cong \tau^{m}$ on $\mathcal{C}$ and $\tau \cong \Omega^{2} \cong \tau^{2 m}$.

2.2. Suppose $\mathcal{C}$ is a quasi-serial stable component and $M=[r] X$ belongs to $\mathcal{C}$. Modifying [R2 (3.3)] slightly, the full subquiver $\mathcal{W}(M)$ of $\mathcal{C}$ whose vertices are given by $\tau^{l}([s] X)$ with $1 \leq s \leq r$ and $0 \leq l \leq r-s$ is called the wing spanned by $M$.

Suppose $\mathcal{W}(X(r+1))$ is a wing and $W$ is an indecomposable module with $W, \Omega^{-} W \notin \mathcal{W}\left(\tau^{-} X(r)\right)$. As in $1.5 d_{W}$ denotes the function $\operatorname{dim} \underline{\operatorname{Hom}}(W,-)$. Then we have

$$
d_{W}(X(r+1))=\sum_{i=0}^{r} d_{W}\left(\tau^{-i} X\right) .
$$

Consider the Auslander-Reiten sequence

$$
0 \rightarrow X(r) \rightarrow X(r+1) \oplus \tau^{-} X(r-1) \oplus P \rightarrow \tau^{-} X(r) \rightarrow 0
$$

where $P$ is projective or zero. Then $d_{W}(X(r+1))=d_{W}(X(r))+d_{W}\left(\tau^{-} X(r)\right)-$ $d_{W}\left(\tau^{-} X(r-1)\right)$ by 1.5.1. The claim follows by induction on $r$.

2.2.1. We call a full subquiver of a quasi-serial component $\mathcal{C}$ a ladder if its vertices are given by all $[s] X$ for $1 \leq s \leq r$ together with $\tau[t] X$ for $1 \leq t<r$ (or dually by the vertices $X(s)$ for $1 \leq s \leq r$ together with $\tau^{-} X(t)$ for $1 \leq t<r$.

Lemma. Given any ladder, there is at most one projective attached to its interior:

Proof. Assume for some $m>1$ there is an Auslander-Reiten sequence in $\mathcal{C}$, say

$$
0 \rightarrow \tau X(m) \stackrel{\epsilon}{\rightarrow} \tau X(m+1) \oplus P \oplus X(m-1) \stackrel{\pi}{\rightarrow} X(m) \rightarrow 0
$$


where $P$ is indecomposable projective and where $\epsilon=\left(\epsilon_{i}\right)$ and $\pi=\left(\pi_{i}\right)^{t}$. We will show that $P$ is the unique indecomposable projective in the ladder starting at $\tau X(m+1)$ and $X(m)$, respectively $\tau X(m)$ and $\tau X(m+1)$. Then $P \neq 0$ if and only if $\pi_{1}$ and $\pi_{3}$ are mono; moreover, if this happens, then $\epsilon_{1}, \epsilon_{3}$ are epi. If $\epsilon_{3}$ is epi, then it follows by induction on $i$ that all the irreducible maps $\tau X(m-i) \rightarrow X(m-i-1)$ are surjective for $0 \leq i \leq m-2$. Similarly, $\pi_{3}: X(m-1) \rightarrow X(m)$ mono gives irreducible monos $\tau^{-i} X(m-i-1) \rightarrow \tau^{-i} X(m-i)$ for $0 \leq i \leq m-2$, too. Therefore, there is no non-zero projective in the ladder starting with $\tau X(m) \rightarrow X(m-1)$ as well as in the ladder starting at $\tau X(m)$ and $\tau X(m+1)$.

2.3. If $A$ is a representation-infinite hereditary algebra and $\mathcal{C}$ a regular component in the Auslander-Reiten quiver of $A$, then $\mathcal{C}$ is either a tube or of type $\mathbf{Z} A_{\infty}$. All irreducible maps $X(i) \rightarrow X(i+1)$ are injective and one may consider them as inclusions. If $A$ is tame hereditary, then $\mathcal{C}$ is a tube of period $k$ with quasisimple modules $X_{1}, \ldots, X_{k}$. The category add $\mathcal{C}$ is a serial abelian category, that is, any indecomposable object in $\operatorname{add} \mathcal{C}$ has a unique composition series in add $\mathcal{C}$ and the simple objects in this category are the modules $X_{1}, \ldots, X_{k}$; see $\mathrm{R} 2$. This does not hold if $A$ is wild hereditary. But one gets at least that for any quasisimple module $X$ in $\mathcal{C}$ and any natural numbers $i, j$ there is a short exact sequence $0 \rightarrow X(i) \rightarrow X(i+j) \rightarrow \tau^{-i} X(j) \rightarrow 0$; see [R1]. Hence $\mathcal{C}$ was called quasi-serial in this case. The following result explains why we call a component for a self-injective algebra stably quasi-serial, if its stable part is of type $\mathbf{Z} A_{\infty}$ or is a tube.

Proposition. Let $\mathcal{C}$ be a component which is stably quasi-serial, let $X$ be quasisimple in $\mathcal{C}$ and $i, j>0$. Then there exists a short exact sequence

$$
0 \rightarrow X(i) \stackrel{\epsilon}{\rightarrow} X(i+j) \oplus P \stackrel{\pi}{\rightarrow} \tau^{-i} X(j) \rightarrow 0
$$

where $P$ is projective, possibly decomposable or zero, and $\pi=\left(\pi_{1}, \pi_{2}\right)^{t}, \epsilon=\left(\epsilon_{1}, \epsilon_{2}\right)$ with $\epsilon_{1}, \pi_{1}$ chains of irreducible maps corresponding to the sectional paths; and the components of $\pi_{2}, \epsilon_{2}$ are compositions of irreducible maps.

Proof. The proof is done by induction on $i$ and $j$. The first case is formulated as a separate lemma.

2.3.1 Lemma. Let $\mathcal{C}$ be a component which is stably quasi-serial, and let $X(r)=$ $[r] Y$ be indecomposable in $\mathcal{C}$.

(a) There exists a short exact sequence

$$
0 \rightarrow \tau X \stackrel{\epsilon}{\rightarrow} \tau X(r+1) \oplus P \stackrel{\pi}{\rightarrow} X(r) \rightarrow 0
$$

where $\pi=\left(\begin{array}{c}\pi_{1} \\ \pi_{2}\end{array}\right)$ and $\epsilon=\left(\epsilon_{1}, \epsilon_{2}\right)$ with $\pi_{1}$ irreducible, and where $P$ is zero or the indecomposable projective in the ladder starting at $\tau X(r+1), X(r)$ and $\pi_{2}, \epsilon_{i}$ are compositions of irreducible maps. Hence $\epsilon_{1} \pi_{1}$ is in $\mathcal{P}(\tau X, X(r))$.

(b) There exists a short exact sequence

$$
0 \rightarrow X(r) \stackrel{\epsilon}{\rightarrow} X(r+1) \oplus P \stackrel{\pi}{\rightarrow} \tau^{-1} Y \rightarrow 0
$$

where $P$ is zero or the indecomposable projective in the ladder starting at $X(r)$, $X(r+1)$ and where $\epsilon=\left(\epsilon_{1}, \epsilon_{2}\right)$ and $\pi=\left(\begin{array}{l}\pi_{1} \\ \pi_{2}\end{array}\right)$ with $\epsilon_{1}$ irreducible and $\epsilon_{2}, \pi_{i}$ compositions of irreducible maps. Hence $\epsilon_{1} \pi_{1}$ is in $\mathcal{P}\left(X(r), \tau^{-} Y\right)$.

Proof. (a) For $i=1,2, \ldots$ we fix irreducible maps corresponding to Auslander-Reiten sequences which satisfy the mesh relations, as follows. Let

$$
f_{i}: X(i) \rightarrow X(i+1), \quad f_{i}^{\prime}: \tau X(i) \rightarrow \tau X(i+1), \quad g_{i}: \tau X(i+1) \rightarrow X(i) .
$$


If there is a projective attached to the ladder, say between $X(m)$ and $\tau X(m)$, then we have also such irreducible maps $\alpha: \tau X(m) \rightarrow P$ and $\beta: P \rightarrow X(m)$. (This can be done, by using [ARS] V.5.3, V.2.3.)

Take $\epsilon_{1}=f_{1}^{\prime} f_{2}^{\prime} \ldots f_{r}^{\prime}$ and $\pi_{1}=g_{r}$. If there is no projective attached to the ladder, take $\epsilon_{2}=0=\pi_{2}$. Otherwise, take $\epsilon_{2}= \pm f_{1}^{\prime} f_{2}^{\prime} \ldots f_{m-1}^{\prime} \alpha$ and $\pi_{2}=\beta f_{m} f_{m+1} \ldots f_{r-1}$; the mesh relations imply that $\epsilon \pi=0$ for the appropriate choice of the sign.

The map $\epsilon$ is injective by definition. We show that $\pi$ is surjective. This is obvious if $P=0$ or $m=r$, so we assume $P \neq 0$ and we use induction on $r-m$. Assume $m<r$ and $\pi$ is not surjective. Take $x \in X(r) \backslash \operatorname{Im}(\pi)$. Choose $x_{1} \in$ $\tau X(r+1), x_{2} \in X(r-1)$ such that $x_{1} g_{r}+x_{2} f_{r-1}=x$. By induction, the map $\left(\begin{array}{c}g_{r-1} \\ \pi_{2}^{\prime}\end{array}\right): \tau X(r) \oplus P \rightarrow X(r-1)$ is surjective; hence $x_{2}=y g_{r-1}+z \pi_{2}^{\prime}$. But then we get $x=x_{1} g_{r}+y g_{r-1} f_{r-1}+z \pi_{2}=\left(x_{1}+y f_{r}^{\prime}\right) g_{r}+z \pi_{2}$, a contradiction.

From the construction it follows that $\operatorname{dim}(\tau X(r+1) \oplus P)=\operatorname{dim}(\tau X \oplus X(r))$, hence the sequence is exact. Part (b) is proved similarly.

2.3.2. We continue with the proof of Proposition 2.3. We fix the irreducible maps in the relevant part of the component $\mathcal{C}$ such that all mesh-relations are satisfied (this can be done locally; see [ARS] V5.3, V2.3). We denote by $f_{s}: X(i+s-1) \rightarrow$ $X(i+s)$ for $s \geq 1$ the irreducible maps, and we take $\epsilon_{1}=f_{1} \cdots f_{j}$. Similarly, for $\pi_{1}$ we take the composition of the irreducible maps corresponding to the sectional path $X(i+j) \rightarrow \tau^{-i} X(j)$.

Consider the set of indecomposable projective modules $\left\{P_{1}, \ldots, P_{r}\right\}$ such that there are paths $\alpha_{t}$ from $X(i)$ to $P_{t}$ and $\beta_{t}$ from $P_{t}$ to $\tau^{-i} X(j)$ in $\mathcal{C}$ and the composed path has length $i+j$. We may additionally assume that there are no further projective modules on the composed path $\alpha_{t} \beta_{t}$. Let $e_{t}: X(i) \rightarrow P_{t}$ and $p_{t}: P_{t} \rightarrow$ $\tau^{-i} X(j)$ be the chain of irreducible maps corresponding to the chosen paths $\alpha_{t}$ and $\beta_{t}$, define $P=\bigoplus P_{t}$ and $\epsilon_{2}=\left( \pm e_{1}, \ldots, \pm e_{r}\right)$ and $\pi_{2}=\left(p_{1}, \ldots, p_{r}\right)^{t}$.

First, observe the following. If $\gamma$ is a path from $X(i)$ to $\tau^{-i} X(j)$ such that no projective occurs in the part of $\mathcal{C}$ between $\gamma$ and the sectional paths $X(i) \rightarrow \tau^{-i+1} X$ and $\tau^{-i} X \rightarrow \tau^{-i} X(j)$, then any composition of irreducible maps corresponding to $\gamma$ is zero. Using this and the mesh relations one sees that there is a choice of signs such that $\epsilon \pi=0$. (See Figure 1.)

Since $\operatorname{dim}(X(i+j) \oplus P)=\operatorname{dim}\left(X(i) \oplus \tau^{-i} X(j)\right)$, it suffices to show that $\epsilon$ is injective and $\pi$ is surjective.

Consider the ladder starting at $X(i)$ and $X(i+1)$. If no projective module is in the interior of the ladder, clearly $f_{1}$ is injective and additionally we may assume that all the maps $e_{i}$ are of the form $f_{1} e_{i}^{\prime}$. Since $i+j=(i+1)+(j-1)$ we may assume by induction that $\epsilon^{\prime}=\left(f_{2} \cdots f_{i}, e_{1}^{\prime}, \ldots e_{r}^{\prime}\right): X(i+1) \rightarrow X(i+j) \oplus P$ is injective. But $\epsilon=f_{1} \epsilon^{\prime}$ in this case.

If there is a projective occurring in the ladder, then by 2.2 .1 there is only one, $P_{1}$ say; and by 2.3.1(b) the map $\left(f_{1}, e_{1}\right): X(i) \rightarrow X(i+1) \oplus P_{1}$ is injective. For $j>1$ we may assume $e_{j}=f_{1} e_{j}^{\prime}$. Again, we get by induction that the map $\left(f_{2} \cdots f_{j}, e_{2}^{\prime}, \ldots, e_{r}^{\prime}\right): X(i+1) \rightarrow X(i+j) \oplus\left(\bigoplus_{j=2}^{r} P_{j}\right)$ is injective. Hence $\epsilon$ is injective as well.

Dually one shows the surjectivity of $\pi$, using 2.3.1(a) and induction.

2.4 Lemma. Assume $\mathcal{C}$ is a quasi-serial regular component and $X, Y$ in $\mathcal{C}$ are quasi-simple with $X(r) \cong[r] Y$. 


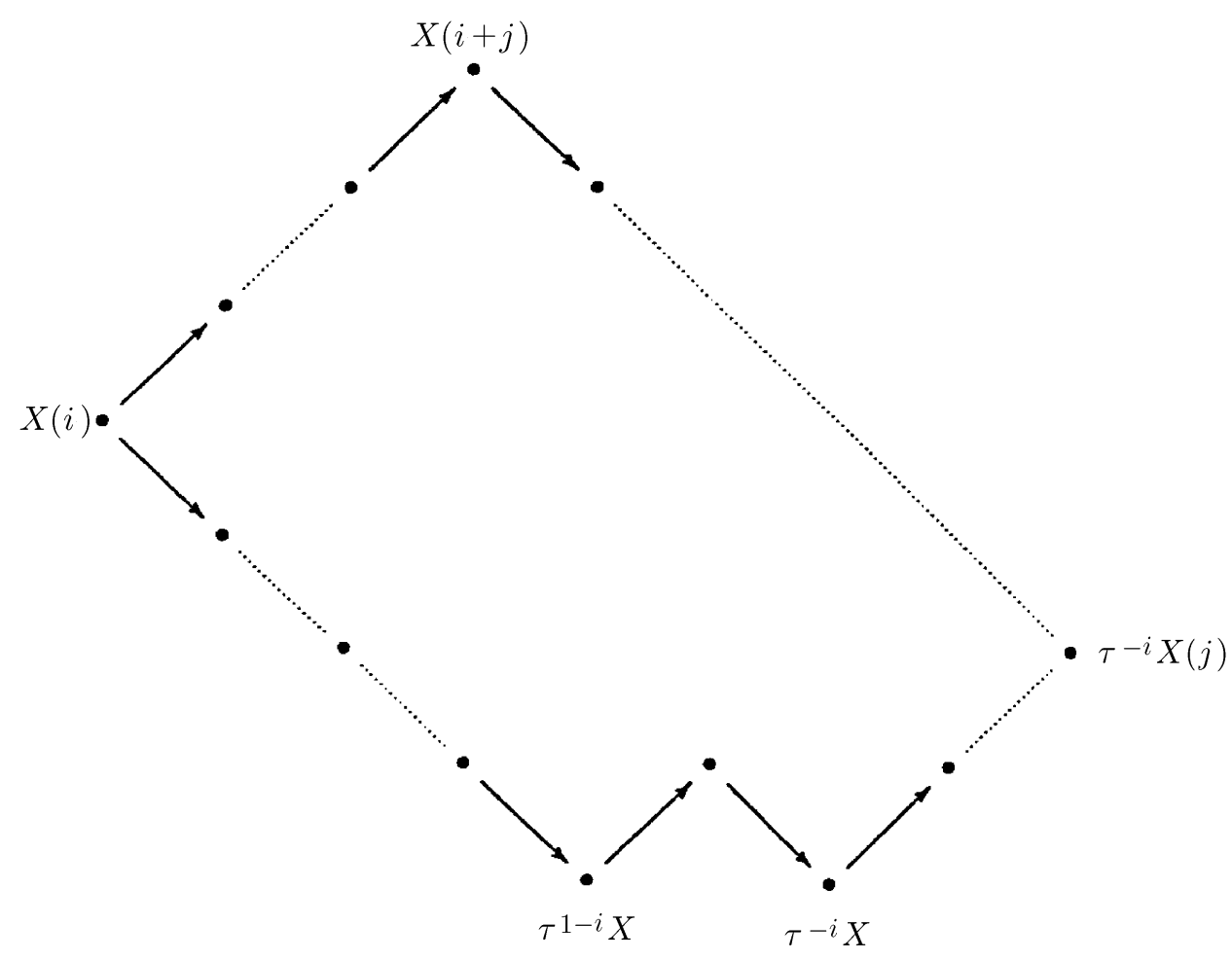

FIGURE 1.

(a) Suppose $\pi:[r+1] Y \rightarrow[r] Y$ is an irreducible epi. If $W$ is indecomposable and not isomorphic to $X(s)$ for $1 \leq s \leq r$, then $\pi$ induces an epimorphism

$$
\operatorname{Hom}(W,[r+1] Y) \rightarrow \operatorname{Hom}(W,[r] Y) \rightarrow 0 .
$$

(b) Suppose $\epsilon: X(r) \rightarrow X(r+1)$ is an irreducible mono. If $W$ is indecomposable and not isomorphic to $[s] Y$ for $1 \leq s \leq r$, then $\epsilon$ induces an epimorphism

$$
\operatorname{Hom}(X(r+1), W) \rightarrow \operatorname{Hom}(X(r), W) \rightarrow 0 .
$$

This is proved in $[\mathrm{R} 1$; the proof given there does not use that the algebra is hereditary. In fact, it holds for an arbitrary finite-dimensional algebra.

The hypotheses in 2.4 are also necessary. With the notation as in 2.4(a), consider $M=X(s)$ where $1 \leq s \leq r$, then the map $(M, \pi)$ is not surjective. If $r=s$, then $i d_{M}$ does not factor through $\pi$. Moreover, for $1 \leq s<r$ there is a monomorphism $\epsilon: M \rightarrow X(s)$ which is a composition of irreducible maps. Then $\epsilon$ does not factor through $\pi$. (Suppose $\epsilon=\psi \pi$ for $\psi: M \rightarrow[r+1] Y$. By applying 2.4(b) repeatedly we see that $\epsilon$ induces an epimorphism

$$
\operatorname{Hom}(X(s),[r+1] Y) \rightarrow \operatorname{Hom}(X(r),[r+1] Y) \rightarrow 0 .
$$

Hence $\psi=\epsilon \eta$ for $\eta: X(s) \rightarrow[r+1] Y$. Now $\epsilon=\epsilon \eta \pi$; since $1-\eta \pi$ is an isomorphism, it follows that $\epsilon=0$, a contradiction.)

Similarly, if $M=[s] Y$ for some $s$ with $1 \leq s \leq r$, then $(\epsilon, M)$ is not surjective. 
2.4.1. Lemma 2.4 has a stable version.

Lemma. Assume $\mathcal{C}$ is a stably quasi-serial component and $X, Y$ are quasi-simple in $\mathcal{C}$ with $X(r) \cong[r] Y$. Let $W$ be indecomposable.

(a) Suppose $\pi:[r+1] Y \rightarrow[r] Y$ is irreducible. If $W$ is not isomorphic to $X(s)$ for $1 \leq s \leq r$, then $\pi$ induces an epimorphism

$$
\underline{\operatorname{Hom}}(W,[r+1] Y) \rightarrow \underline{\operatorname{Hom}}(W,[r] Y) \rightarrow 0 .
$$

(b) Suppose $\epsilon: X(r) \rightarrow X(r+1)$ is irreducible. If $W$ is not isomorphic to $[s] Y$ for $1 \leq s \leq r$, then $\epsilon$ induces an epimorphism

$$
\underline{\operatorname{Hom}}(X(r+1), W) \rightarrow \underline{\operatorname{Hom}}(X(r), W) \rightarrow 0 .
$$

Proof. (a) There is a module $P$ which is projective or zero and an irreducible map

$$
P \oplus[r+1] Y \stackrel{\pi_{1}}{\rightarrow}[r] Y .
$$

This induces

$$
\underline{\operatorname{Hom}}(W, P \oplus[r+1] Y) \stackrel{\overline{\pi_{1}}}{\rightarrow} \underline{\operatorname{Hom}}(W,[r] Y) .
$$

We proceed by induction on $r$. If $r=1$, then $P \oplus[r+1] Y$ is the whole middle term of the Auslander-Reiten sequence and then $\left(W, \pi_{1}\right)$ is surjective and hence $\bar{\pi}_{1}$ is surjective, by 1.4 .

Now assume $r>1$. Let $f: W \rightarrow[r] Y$, then $f$ is not a split epimorphism, so we have $f=g_{1} \pi_{1}+g_{2} \pi_{2}$ where the Auslander-Reiten sequence is given by

$$
0 \rightarrow \tau[r] Y \stackrel{\left(\epsilon_{1}, \epsilon_{2}\right)}{\longrightarrow}(P \oplus[r+1] Y) \oplus \tau[r-1] Y \stackrel{\left(\begin{array}{l}
\pi_{1} \\
\pi_{2}
\end{array}\right)}{\longrightarrow}[r] Y \rightarrow 0 .
$$

Note that $\epsilon_{1} \pi_{1}+\epsilon_{2} \pi_{2}=0$.

Consider the irreducible map $\epsilon_{2}$. By the inductive hypothesis, $\epsilon_{2}$ induces a surjective map

$$
\underline{\operatorname{Hom}}(W, \tau[r] Y) \rightarrow \underline{\operatorname{Hom}}(W, \tau[r-1] Y) \rightarrow 0 .
$$

This gives $\underline{g_{2}}=\underline{\eta \epsilon_{2}}$ for $\eta: W \rightarrow \tau[r] Y$, that is,

$$
g_{2}=\eta \epsilon_{2}+\rho, \quad \rho \in \mathcal{P}(W, \tau[r] Y)
$$

and therefore $f=g_{1} \pi_{1}+\left(\eta \epsilon_{2}+\rho\right) \pi_{2}=\left(g_{1}-\eta \epsilon_{1}\right) \pi_{1}+\rho \pi_{2}$ and $\underline{f}=\underline{h \pi_{1}}$ for some $h$, as required.

Part (b) is similar.

2.5. We combine $1.4,2.3$ and 2.4, 2.4.1 and obtain the following:

(a) Assume $0 \rightarrow \tau X \stackrel{\epsilon}{\rightarrow} \tau X(r+1) \oplus P \stackrel{\pi}{\rightarrow} X(r) \rightarrow 0$ is the short exact sequence as in 2.3.1(a) and let $X(r)=[r] Y$.

Suppose $W$ is indecomposable, then we have an exact sequence

$$
\underline{\operatorname{Hom}}(W, \tau X) \stackrel{\bar{\epsilon}}{\rightarrow} \underline{\operatorname{Hom}}(W,[r+1] Y) \stackrel{\bar{\pi}}{\rightarrow} \underline{\operatorname{Hom}}(W,[r] Y) .
$$

(1) If $\Omega^{-1} W \approx X(s)$ for $1 \leq s \leq r$, then $\bar{\epsilon}$ is $1-1$.

Namely, by 2.4 .1 we know $\underline{\operatorname{Hom}}\left(\Omega^{-} W, \pi\right)$ is onto, and then by $1.4(2)$ also $\left(\Omega^{-1} W, \pi\right)$ is onto. Now 1.4(1) implies that $\bar{\epsilon}$ must be $1-1$.

(2) If $W \neq X(s)$ for $1 \leq s \leq r$, the $\bar{\pi}$ is onto.

Hence, if $W, \Omega^{-1} W \neq X(s)$ for $1 \leq s \leq r$, then

$$
d_{W}(\tau X)+d_{W}([r] Y)=d_{W}([r+1] Y) .
$$

(b) Assume $0 \rightarrow X(r) \stackrel{\epsilon}{\rightarrow} X(r+1) \oplus P \stackrel{\pi}{\rightarrow} \tau^{-1} Y \rightarrow 0$ is exact, as in 2.3.1(b) 
Suppose $W$ is indecomposable, then we have an exact sequence

$$
\underline{\operatorname{Hom}}\left(\tau^{-1} Y, W\right) \stackrel{\bar{\pi}}{\rightarrow} \underline{\operatorname{Hom}}(X(r+1), W) \stackrel{\bar{\epsilon}}{\rightarrow} \underline{\operatorname{Hom}}(X(r), W) .
$$

(1) If $\Omega W \not[s] Y$ for $1 \leq s \leq r$, then $\bar{\pi}$ is $1-1$.

(2) If $W \neq[s] Y$ for $1 \leq s \leq r$, then $\bar{\epsilon}$ is onto.

In particular, if $W, \Omega W \neq[s] Y$ for $1 \leq s \leq r$, then

$$
d_{W}^{\prime}\left(\tau^{-} Y\right)+d_{W}^{\prime}(X(r))=d_{W}^{\prime}(X(r+1)) .
$$

2.6 Lemma. Let $\mathcal{C}$ be a stably quasi-serial component. If $[r] X \in \mathcal{C}$ has quasilength $r$ and quasi-top $X$, then a chain of irreducible maps $\pi:[r] X \rightarrow[s] X$ $\left(\epsilon: \tau^{r-s}[s] X \rightarrow[r] X\right.$, respectively) for $r>s \geq 1$ does not factor through a projective module.

Proof. We may assume that $s=1$. First, it will be shown that $\underline{\pi}=0$ implies $\underline{\operatorname{Hom}}([r] X, X)=0$. For $0 \neq \underline{f} \in \underline{\operatorname{Hom}}([r] X, X)$ we get $\underline{f}=\underline{g \pi}$ by 2.4 .1 , with $g \in \operatorname{End}([r] X)$, hence $\underline{\pi} \neq 0$.

Suppose next $\underline{\operatorname{Hom}}([r] X, X)=0$. By 2.2 we have

$$
0<\operatorname{dim} \underline{\operatorname{End}}([r] X)=d_{[r] X}([r] X)=\sum_{i=0}^{r-1} d_{[r] X}\left(\tau^{i} X\right) .
$$

Hence we get $d_{[r] X}\left(\tau^{i} X\right) \neq 0$ for some $i$ and from the assumption $i>0$. Let $0 \neq f \in \underline{\operatorname{Hom}}\left([r] X, \tau^{i} X\right)$. Again, by 2.4.1 we deduce the existence of a map $g \in \overline{\operatorname{Hom}}\left([r] X, \tau^{i}[r] X\right)$ with $\underline{f}=g \pi^{\prime}$, where $\pi^{\prime}: \tau^{i}[r] X \rightarrow \tau^{i} X$ is a chain of irreducible maps. But $\underline{\pi^{\prime}}=0$ gives the contradiction. Dually one shows $\underline{\epsilon} \neq 0$.

2.6.1 Corollary. In 2.4.1 the converse also is true: With the notation as in 2.4.1 we have:

(a) If $W \cong X(s)$ for some $s$ with $1 \leq s \leq r$, then the map induced by $\pi$

$$
\underline{\operatorname{Hom}}(W,[r+1] Y) \rightarrow \underline{\operatorname{Hom}}(W,[r] Y)
$$

is not surjective.

(b) If $W \cong[s] Y$ for some $s$ with $1 \leq s \leq r$, then the map induced by $\epsilon$

$$
\underline{\operatorname{Hom}}(X(r+1), W) \rightarrow \underline{\operatorname{Hom}}(X(r), W)
$$

is not surjective.

Proof. (a) Let $f=i d$ if $r=s$ and $f=\epsilon_{1}: W \rightarrow[r] Y$ the sectional chain of irreducible maps, otherwise. We claim that $\underline{f}$ does not lie in the image of the map induced by $\pi$. Otherwise, we would have

$$
f=\psi \pi+\rho, \quad \text { for } \psi \in \operatorname{Hom}(W,[r+1] Y) \rho \in \mathcal{P}(W,[r] Y) .
$$

In case $f=i d$ we have $\rho$ is in the radical of $\operatorname{End}([r] Y)$, hence is nilpotent and $\pi$ splits, a contradiction.

Now suppose $f=\epsilon_{1}$. By applying 2.4.1(b) repeatedly we have that the map

$$
\overline{\epsilon_{1}}: \underline{\operatorname{Hom}}(X(r),[r+1] Y) \rightarrow \underline{\operatorname{Hom}}(X(s),[r+1] Y)
$$

is surjective. So $\psi=\epsilon_{1} \eta+\rho_{1}$ with $\rho_{1} \in \mathcal{P}(W,[r+1] Y)$. It follows that

$$
\epsilon_{1}=\left(\epsilon_{1} \eta+\rho_{1}\right) \pi+\rho
$$

and $\epsilon_{1}(1-\eta \pi)$ factors through a projective. Since $1-\eta \pi$ is a unit, $\epsilon_{1}$ factors through a projective module, a contradiction to 2.6. Part (b) is similar. 


\section{ON DIMENSIONS OF SPACES OF STABLE HOMOMORPHISM}

3.1. In this section, $\Lambda$ is an arbitrary finite-dimensional represenation infinite connected self-injective algebra, and we assume that $f$ is some equivalence of the stable category $\underline{\bmod } \Lambda$; this includes $f=\tau^{s}$ or $f=\Omega^{s}$. We study the function

$$
\psi_{f}(M)=\operatorname{dim} \underline{\operatorname{Hom}}_{\Lambda}(M, f M)
$$

for $M$ a module. Since $f$ commutes with $\tau, \Omega$ (see ARS] X.1) it is constant on $\tau$-orbits and on $\Omega$-orbits.

3.2 Lemma. Given an exact sequence in some quasi-serial component

$$
0 \rightarrow \tau[r-1] Z \stackrel{\epsilon}{\rightarrow}[r] Z \oplus P \stackrel{\pi}{\rightarrow} Z \rightarrow 0
$$

with $Z$ quasi-simple, as in 2.3.1. Suppose $W$ is indecomposable such that neither $W$ nor $\Omega W$ is a module in the same component of quasi-length $<r$, then $d_{W}^{\prime}$ is additive on such an exact sequence.

In particular, for $W=f[r] Z$ we get

$$
d_{\tau[r-1] Z}(f[r] Z) \leq \psi_{f}([r] Z)
$$

Proof. The first part follows from 1.4.1. For the second part $W=f[r] Z$ has quasilength $r$ and by the first part $d_{W}^{\prime}(\tau[r-1] Z) \leq d_{W}^{\prime}([r] Z)$. Now, $d_{W}^{\prime}(\tau[r-1] Z)=$ $d_{\tau[r-1] Z}(W)=d_{\tau[r-1] Z}(f[r] Z)$ and $d_{W}^{\prime}\left(([r] Z)=\psi_{f}(([r] Z)\right.$.

3.3 Theorem. Let $\mathcal{C}$ be a stable component which is quasi-serial and assume that it is not the case that $\tau \Omega^{-1} \cong f \neq \tau$ on $\mathcal{C}$. Suppose that $M, Q \in \mathcal{C}$ and $q l(Q)<q l(M)$, then

$$
\psi_{f}(Q) \leq \psi_{f}(M)
$$

Proof. Let $r=q l(M)$; it suffices to consider the case when there is an irreducible map $Q \rightarrow M$ and $q l(Q)=r-1$. Let $Y$ be the quasi-top of $f M$, then $f M=[r] Y$ and $f Q=\tau[r-1] Y$. We consider the values of the function $d_{Q}$ on the ladder starting with $f Q \rightarrow f M$ and ending at $Y$. Let

$$
a_{i}=d_{Q}([i] Y), \quad 1 \leq i \leq r, \text { and } b_{i}=d_{Q}(\tau[i] Y), \quad 1 \leq i \leq r-1
$$

Then $b_{r-1}=d_{Q}(f Q)=\psi_{f}(Q)$ and moreover, we have $a_{r}=d_{Q}(f M)$ and hence by 3.2 we know that $a_{r} \leq \psi_{f}(M)$. So it suffices to show that $b_{r-1} \leq a_{r}$.

By considering quasi-lengths one sees that $Q, \Omega^{-1} Q$ cannot occur in the lower part of the ladder and hence $d_{Q}$ is additive on this part. Therefore,

$$
b_{1}+a_{1}=a_{2}, \quad b_{i}+a_{i}=a_{i+1}+b_{i-1}, 2 \leq i \leq r-2 .
$$

This implies

$$
b_{i}=a_{i+1}-a_{1}, \quad 1 \leq i \leq r-2 .
$$

Case 1 . Assume $f \neq \tau$ on $\mathcal{C}$, then by the hypothesis also $f \neq \tau \Omega^{-1}$ on $\mathcal{C}$. Hence by $1.5 .1, d_{Q}$ is additive on the whole ladder and

$$
b_{r-1}+a_{r-1}=a_{r}+b_{r-2} .
$$

It follows that $b_{r-1}=a_{r}-a_{1} \leq a_{r} \leq \psi_{f}(M)$, as required.

Case 2. Assume $f \cong \tau$ on $\mathcal{C}$. Then the remaining Auslander-Reiten sequence of the ladder is of the form

$$
0 \rightarrow \tau Q \stackrel{\epsilon}{\rightarrow} \tau M \oplus M^{\prime} \stackrel{\pi}{\rightarrow} Q \rightarrow 0
$$


(where $M^{\prime}=\tau[r-2] Y \oplus P$ for $P$ projective or zero; for $r=2$ the first summand of $M^{\prime}$ is zero). By 1.5.2 this induces an exact sequence

$$
\eta: \underline{\operatorname{Hom}}(Q, f Q) \stackrel{\bar{\epsilon}}{\rightarrow} \underline{\operatorname{Hom}}\left(Q, f M \oplus M^{\prime}\right) \rightarrow \underline{\operatorname{rad}}(Q, Q) \rightarrow 0 .
$$

Assume first that $Q \neq \Omega(Q)$. Then $\left(\Omega^{-1} Q, \pi\right)$ is onto and by 1.4 we have that $\bar{\epsilon}$ is 1-1 and

$$
a_{r}+b_{r-2}=b_{r-1}+\left(a_{r-1}-1\right)
$$

hence $a_{r}-b_{r-1}=a_{1}-1$. Now, $a_{1}=\operatorname{dim} \underline{\operatorname{Hom}}(Q, Y)$ here and this is non-zero by 2.6. Therefore, $a_{r}-b_{r-1} \geq 0$, as required.

Now assume $Q \cong \Omega(Q)$. Then in the above exact sequence $\eta$, the kernel of $\bar{\epsilon}$ has dimension 1. So we get $a_{r}-b_{r-1}=a_{1}-2$. As before, we know that $a_{1} \geq 1$ and hence $b_{r-1}=\psi_{f}(Q) \leq a_{r}+1$. If we can show that $a_{r}<\psi_{f}(M)$, then we are done. From 3.2 we know that

$$
a_{r}+\operatorname{dim} \underline{\operatorname{Hom}}(Z, f M)=\psi_{f}(M)
$$

where $Z$ is the quasi-top of $M$. So we must show that

$$
\underline{\operatorname{Hom}}(Z, f M) \neq 0 \text {. }
$$

Since $f \cong \tau$ and $\Omega \cong i d$ on $\mathcal{C}$ we have $K$-isomorphisms

$$
\underline{\operatorname{Hom}}(Z, f M) \cong \operatorname{Ext}^{1}(M, Z) \cong \underline{\operatorname{Hom}}(M, Z) \neq 0
$$

again by 2.6 .

3.3.1. Consider a quasi-serial component $\mathcal{C}$ such that $\tau \Omega^{-1} \cong f \neq \tau$ on $\mathcal{C}$.

(1) Suppose $f$ fixes $\mathcal{C}$, then this is quite exceptional. Namely, $f$ must fix each $\tau$-orbit in $\mathcal{C}$ and hence there is some integer $s$ such that

$$
\tau \Omega^{-1} M \cong f M \cong \tau^{s} M \quad(M \in \mathcal{C})
$$

Since $\tau \cong \Omega^{2} \nu$ where $\nu$ is a Nakayama twist, it follows that $\Omega^{2 s-1} \cong \nu^{1-s}$ on $\mathcal{C}$. This preserves dimensions of modules. So if this happens, then all modules in $\mathcal{C}$ have bounded projective resolutions. If in addition $\Lambda$ is symmetric, then $\nu \cong i d$. It is easy to see that $\tau^{s-1} \cong \Omega^{-1} \not i d$ on $\mathcal{C}$ if and only if $\mathcal{C}$ is a tube fixed by $\Omega$ of rank $t>1$ where $t$ divides $2 s-1$.

(2) Similarly, if $f=\Omega^{s}$, then it follows that $\nu \cong \Omega^{s-1}$ on $\mathcal{C}$ and modules in this component have bounded projective resolutions. In case $\Lambda$ is symmetric we have $\mathcal{C}$ must be a tube of rank $t$ and $t$ divides $s-1$.

3.3.2. In general, it is possible that $q l(Q)<q l(M)$ but $\psi_{f}(Q)=\psi_{f}(M)$. An example can be found in [EKS, 6.6] with $f=i d$ : for any natural number $n \geq 2$ there exists a symmetric algebra $A$ with $n$ simple modules which has a component $\mathcal{C}$ of the form $\mathbf{Z} A_{\infty}$ containing stable bricks of quasi-length $n-1$. Hence we have $\psi_{f}(Q)=1$ for all $Q \in \mathcal{C}$ with $q l(Q) \geq n-1$.

3.4. In the situation of 3.3, we have a vanishing condition: Suppose $q l(Q)+1=$ $q l(M)$ and there is an irreducible map $Q \rightarrow M$. Assume we are in Case 1 of the proof. If $\psi_{f}(Q)=\psi_{f}(M)$, then $d_{Q}(Y)=0$ where $Y$ is the quasi-top of $f M$. 
3.5. One may ask whether the function $\psi_{f}$ is unbounded on a quasi-serial component. For tubes we get.

Lemma. Let $\mathcal{T}$ be a tube, and let $f$ be a stable equivalence of $\underline{\bmod } \Lambda$. Then the set $\left\{\psi_{f}(M): M \in \mathcal{T}\right\}$ is either unbounded, or $\psi_{f}(M)=0$ for all $M \in \mathcal{T}$. In the latter case we have $f \mathcal{T} \neq \mathcal{T}$.

Proof. Let $\mathcal{T}$ be a tube and suppose $k$ is the period of $\mathcal{T}$.

Assume first $f \mathcal{T}=\mathcal{T}$. Then $f=\tau^{s}$ on the objects of $\mathcal{T}$, for some $s$; see ARS X.1. Let $r \geq m k$ and $M$ be an indecomposable module in $\mathcal{T}$ of quasi-length $r$. If $f M=\tau^{s} M=X(r)$ with quasi-socle $X$, then we have

$$
\psi_{f}(M)=d_{M}\left(\tau^{s} M\right)=d_{M}(X(r)) .
$$

Clearly $M, \Omega^{-} M$ do not belong to $\mathcal{W}\left(\tau^{-} X(r-1)\right)$, hence by 2.2 we have

$$
d_{M}(X(r))=\sum_{i=0}^{r-1} d_{M}\left(\tau^{-i} X\right) .
$$

Let $\delta=\sum_{i=0}^{k-1} d_{M}\left(\tau^{-i} X\right)$, then $\delta$ is non-zero (since the quasi-top of $M$ occurs amongst the modules $\left.\tau^{-i} X\right)$. It follows that $d_{M}(X(r)) \geq m \delta \geq m$.

Suppose $f \mathcal{T} \neq \mathcal{T}$ and there is some indecomposable $M=Y(r)$ in $\mathcal{T}$ with $\psi_{f}(M) \neq 0$. Let $f M=X(r)$. Again, we get

$$
\psi_{f}(M)=\sum_{i=0}^{r-1} d_{M}\left(\tau^{-i} X\right)
$$

therefore $\delta=\sum_{i=0}^{k-1} d_{M}\left(\tau^{-i} X\right)$ is non-zero. From (2.4.1(b)) we get $d_{Y(r)}\left(\tau^{-i} X\right) J \leq$ $d_{Y(r+k)}\left(\tau^{-i} X\right)$. Hence we have $\psi_{f}(Y(r+k)) \geq \psi_{f}(M)+\delta$ and the claim follows.

3.5.1. In the special case when $f=i d$ we get

Lemma. Let $\mathcal{T}$ be a tube of period $k$. If $M$ is indecomposable in $\mathcal{T}$ with quasi-length $r=m k+s$ with $1 \leq s<k$, then $\operatorname{dim} \underline{\operatorname{End}}(M) \geq m+1$.

Proof. Let $X$ be the quasi-top of $M$. By 2.2 we have $\operatorname{dim} \underline{\operatorname{End}}(M)=\sum_{i=0}^{r-1} d_{M}\left(\tau^{i} X\right)$ $\geq(m+1) d_{M}(X)$. But $d_{M}(X)>0$ by 2.6 .

3.6 Lemma. Let $\mathcal{C}$ be a stable component which is isomorphic to $\mathbf{Z} A_{\infty}$ which is not fixed by $\Omega$, and let $f=\tau^{s}$. If $\left\{\psi_{f}(M): M \in \mathcal{C}\right\}$ is bounded, then for $m \gg 0$

$$
\underline{\operatorname{Hom}}\left(X, \tau^{-m} X\right)=0=\underline{\operatorname{Hom}}\left(\tau^{m} X, X\right)
$$

where $X \in \mathcal{C}$ is quasi-simple.

Proof. Since $\mathcal{C}$ is not fixed by $\Omega$, the hypothesis for 3.3 is satisfied. By 3.3 there is some $r_{0}$ such that for all $Z \in \mathcal{C}$ with $q l(Z) \geq r_{0}$ we have

$$
\psi_{f}(Z)=d
$$

Fix a quasi-simple module $X$ in $\mathcal{C}$ and set $Y:=\tau^{s} X$. We will first show that

$$
\text { for all } r \geq r_{0}, \quad d_{Y}^{\prime}([r] \tau X)=0 .
$$

Let $r \geq r_{0}$. Clearly, $\tau^{s-1} \neq i d$ on $\mathcal{C}$; we apply 3.4 with $Q=\tau[r] X$ (and $M=$ $[r+1] X)$, and we get $d_{Q}(Y)=0$; but $d_{Q}(Y)=d_{Y}^{\prime}(Q)$.

Let $r_{1}=\max \left\{r_{0}, s+1\right\}$ and let $\left(\rightarrow\left[r_{1}\right] \tau X\right)$ be the cone of all predecessors of $\left[r_{1}\right] \tau X$ in $\mathcal{C}$. The function $d_{Y}^{\prime}$ is additive on this cone by 1.5.1. 
For $a=1,2,3, \ldots, r_{1}$ we set $k_{a}:=d_{Y}^{\prime}\left(\left[r_{1}-a+1\right] \tau^{a} X\right)$. We claim that

$$
d_{Y}^{\prime}\left([r] \tau^{a} X\right)=k_{a}, \text { constant, for } r \geq r_{1}-a+1 \text {. }
$$

For $a=1$ we have $k_{1}=0$ and the statement was just proved; the general case follows by induction on $a$, by additivity. But then, again by the additivity of $d_{Y}^{\prime}$ on the cone $\left(\rightarrow\left[r_{1}\right] \tau X\right)$ we get

$$
d_{Y}^{\prime}\left([t] \tau^{b} X\right)=0 \text { for all } t \geq 1 \text { and } b>r_{1} .
$$

Hence $\underline{\operatorname{Hom}}\left(X, \tau^{-m} X\right)=0$ for all large $m$, and the statement follows.

3.6.1. We do not know an example of a stable component of type $\mathbf{Z} A_{\infty}$ on which $\psi_{f}$ is bounded and we expect that this may not occur.

The converse of 3.6 is not true. For example, let $H$ be a connected wild hereditary algebra, $\mathcal{D}$ a regular component in $\Gamma_{H}$ and $Y$ quasi-simple in $\mathcal{D}$. Then we have $\operatorname{Hom}\left(Y, \tau^{-m} Y\right)=0$ for $m \gg 0$, by $\mathrm{K} 1$. It follows from Ba that for any integer $s$ the sequence ( $\left.\operatorname{dimHom}\left(Y(m), \tau^{s} Y(m)\right)\right)_{m}$ grows exponentially. If $A$ is the trivial extension of $H$ by $D(H)$ (or more generally a self-injective algebra of wild tilted type as studied in [EKS]), then it follows that the same holds for the stable homomorphisms of $A$ for any stable component of $\mathbf{Z} A_{\infty}$ of $A$ (see [EKS], section 4).

3.7. The Ext-algebra $\operatorname{Ext}^{*}(X, X)$ of a module $X$ is of central interest. As an application of 3.3 we get for the dimensions of the spaces $\operatorname{Ext}^{i}(X, X)$.

Corollary. Let $\mathcal{C}$ be a stable component which is quasi-serial. Suppose $M, Q$ are in $\mathcal{C}$ with $q l(Q)<q l(M)$.

(a) We have $\operatorname{dim} \underline{\operatorname{End}}(Q) \leq \operatorname{dim} \underline{\operatorname{End}}(M)$ unless possibly $\tau \Omega^{-1} \cong i d ¥ \tau$ on $\mathcal{C}$.

(b) We have $\operatorname{dim} \operatorname{Ext}^{s}(Q, Q) \leq \operatorname{dim}_{\operatorname{Ext}^{s}}(M, M)$ for $s \geq 1$, unless possibly $\tau \Omega^{-1} \cong \Omega^{s} \approx \tau$ on $\mathcal{C}$.

Proof. For part (a), take $f=i d$ in 3.3. In (b) take $f=\Omega^{s}$, recall that $\operatorname{Ext}^{s}(M, M)$ $\cong \underline{\operatorname{Hom}}\left(M, \Omega^{s} M\right)$.

3.8. A module whose endomorphism ring is $K$ usually is called a brick. We call an indecomposable module $M$ a stable brick if $\underline{\operatorname{End}}(M)=K$. For example, any $\tau$-translate of a simple non-projective module is a stable brick. Although a stable brick is not normally a brick, we prefer this terminology. We get from 3.7

Corollary. Let $\mathcal{C}$ be a quasi-serial component such that it is not the case that $\tau^{-} \cong \Omega^{-1} \neq i$ on $\mathcal{C}$. Assume $M=X(r)$ is a stable brick in $\mathcal{C}$, of quasi-length $r$. Then for $1 \leq s \leq r$ the modules $X(s)$ are stable bricks.

\section{Applichtions to Group algebras}

4.1. Assume that $\Lambda=K G$ where $G$ is a finite group. Then $\Lambda$ and, more generally, any block $B$ of $\Lambda$ is a symmetric algebra, and our results may be applied. We may assume $\Lambda$ is not semi-simple, that is, the characteristic of $K$ divides the order or $G$. When $G$ is a $p$-group we have, in fact, strict inequality in 3.3 .

4.2 Theorem. Let $\Lambda=K G$ where $G$ is a p-group and char $K=p$. Assume $f$ is a stable equivalence of $\Lambda$ and $\mathcal{C}$ is a quasi-serial component of $\Gamma_{s}(\Lambda)$ which is fixed by $f$. Suppose it is not the case that $\Omega \cong f \neq \Omega^{2}$ on $\mathcal{C}$. Suppose $M, Q \in \mathcal{C}$ with $q l(Q)<q l(M)$, then

$$
\operatorname{dim} \underline{\operatorname{Hom}}(Q, f Q)<\operatorname{dim} \underline{\operatorname{Hom}}(M, f M) .
$$


Proof. Recall that $\tau \cong \Omega^{2}$ for $\Lambda$. It follows from 3.3 that the dimensions in question are weakly increasing as functions of the quasi-length. Assume (for a contradiction) that equality holds for $Q, M$ such that there is an irreducible mono $Q \rightarrow M$ in $\mathcal{C}$. By 3.4 we have that $d_{Q}(Y)=0$ where $Y$ is the quasi-top of $f M$. Hence, using a well-known identity

$$
0=\underline{\operatorname{Hom}}_{\Lambda}(Q, Y) \cong \underline{\operatorname{Hom}}_{\Lambda}\left(K, Q^{*} \otimes Y\right)
$$

where $K$ is the trivial module. Now, $\Lambda$ is a local algebra, and it follows that $Q^{*} \otimes Y$ is projective. This is not possible for non-projective modules in the same Auslander-Reiten-component.

Namely, let $V(M)$ denote the variety of a $\Lambda$-module $M$ (see [B] or [C]). The relevant properties are as follows. One has $V(M)=\{0\}$ if and only if $M$ is projective. Moreover, $V(M)=V\left(M^{*}\right)$ and $V(X \otimes Y)=V(X) \cap V(Y)$; and the variety of modules is constant on stable Auslander-Reiten components.

In the case considered we deduce $\{0\}=V\left(Q^{*} \otimes Y\right)=V\left(Q^{*}\right) \cap V(Y)=V(Y)$ and $Y$ is projective, a contradiction.

4.3 Remarks. (1) Recall that the hypothesis of 4.2 excludes precisely the case when $\mathcal{C}$ is a tube fixed by $\Omega$ such that $f \cong \tau^{s}$ on $\mathcal{C}$ and where $\mathcal{C}$ has rank $t>1$ where $t$ divides $2 s-1$ (see 3.3.1). Modules in such a tube have the property that $2 \operatorname{dim} M$ is divisible by the order of $G$. This follows from the projective resolution. Namely, there is an exact sequence

$$
0 \rightarrow M \rightarrow P_{m} \rightarrow \cdots \rightarrow P_{0} \rightarrow M \rightarrow 0
$$

with $m$ even and where $P_{i}$ is free, hence $\operatorname{dim}\left(P_{i}\right)$ is divisible by $|G|$. By the exactness, $2 \operatorname{dim} M=\sum(-1)^{i}\left(\operatorname{dim}\left(P_{i}\right)\right)$.

(2) If $f=i d$, then the conditions in 4.2 are vacuously satisfied.

4.4 Corollary. Let $\Lambda=K G$ where $G$ is a p-group and charK $=p$. Assume that $\Lambda$ is of wild representation type. If $M$ is indecomposable and $\underline{\operatorname{End}}(M) \cong K$, then $M$ is quasi-simple.

Proof. Apply 4.2 with $f=i d$; all components of $\Gamma_{s}(\Lambda)$ are quasi-serial (by [E2]).

4.5. Let $G$ be a $p$-group and $\operatorname{char} K=p$. It follows from 3.3 that $M$ is a stable brick if and only if $M^{*} \otimes M \cong X_{M} \oplus P$ where $P$ is projective and $X_{M}$ has a simple socle and is non-projective.

Following Dade $[\mathrm{D}]$, a $K G$-module $M$ is defined to be endo-trivial if $M \otimes M^{*} \cong$ $K \oplus P$ where $P$ is projective. Hence an endo-trivial module is a stable brick, and we obtain

Corollary. Let $\Lambda=K G$ where $G$ is a p-group and char $K=p$. If $\Lambda$ is of wild type, then every endo-trivial module $M$ has an Auslander-Reiten sequence whose middle term has a unique non-projective summand.

If $p=2$ and $\Lambda=K D$ where $D$ is a dihedral 2-group, then there are endotrivial modules where the middle term of the Auslander-Reiten sequence has two non-projective indecomposable summands.

4.6. If $G$ is a $p$-group, we were not able to find an example of a $K G$-module which is a stable brick but which is not endo-trivial. After asking various people for examples it turned out that Jon Carlson already knew that stable bricks must always be endo-trivial; this will appear in [CR]. 
4.7 Theorem. Assume $\Lambda=K G$ where $G$ is a finite group and $\mathcal{C}$ is quasi-serial, and let $f=\tau^{s}$. Then the set of dimensions $\left\{\psi_{f}(M) \mid M \in \mathcal{C}\right\}$ is unbounded.

Proof. The statement holds by 3.5 for tubes. So we may assume that $\mathcal{C}=\mathbf{Z} A_{\infty}$. Then $\mathcal{C}$ is not fixed by $\Omega$ (see 2.1). Assume (for a contradiction) that the dimensions are bounded; then it follows from 3.6 that for $m \gg 0$ and $X \in \mathcal{C}$ quasi-simple we have

$$
\underline{\operatorname{Hom}}\left(X, \tau^{-m} X\right)=0 \text {. }
$$

Hence $\operatorname{Ext}^{2 m}(X, X)=0$ for $m \gg 0$. Let $\operatorname{Ext}^{*}(X, X)$ be the Ext-algebra, then it follows that every element in this algebra of positive even degree is nilpotent, and then also every element of positive degree. By [B2, 5.2.3] this happens only when $X$ is projective; and this is a contradiction.

Added in proof. The result mentioned in 4.6 has appeared in Jon F. Carlson, A characterization of endotrivial modules over p-groups, Manuscripta Math. 97 (1998), 303-307. MR 99h:20006

\section{REFERENCES}

[ARS] M. Auslander, I. Reiten, S.O. Smalo, Representation theory of Artin algebras, Cambridge studies in advanced Math. 36 (Cambridge University Press 1995) MR 98e:16011, MR 96c:16015

[Ba] D. BAER, Homological properties of wild hereditary algebras, Representation Theory, Lect. Notes in Mathematics 1177 (Springer, 1986), 1-12 MR 87h:16038

[B] D.J. Benson, Modular representation theory: New trends and methods, Lecture Notes in Mathematics 1081 (Springer, 1984) MR 86g:20013

[B2] D.J. Benson, Representations and cohomology II, Cambridge Studies in Advanced Math. 31 (Cambridge University Press 1991) MR 93g:20099

[BG] K. Bongartz, P. Gabriel, Covering spaces in representation theory, Invent. Math. 65 (1982), 331-378 MR 84i: 16030

[C] J.F. Carlson, Varieties for modules, Arcata Conf. on Representations of Finite Groups, Proc. Symp. Pure Math. 47 (1) (1987), 37-44 MR 89b:20032

[CB] W.W. Crawley-Boevey, On tame algebras and bocses, Proc. London Math. Soc. 56 (1988), 451-483 MR 89c:16028

[CR] J.F. CARLSON, R. Rouquier, Self-equivalences of stable categories of modules over modular group algebras, in preparation.

[D] E.C. DADE, Endo-permutation modules over p-groups, II, Ann. of Math. 108 (1978), 317346 MR 80a:13008b

[EKS] K. Erdmann, O. Kerner, A. Skowroński, Self-injective algebras of wild tilted type; preprint (Bielefeld 97-080)

[ES] K. Erdmann, A. Skowroński, On Auslander-Reiten components of blocks and selfinjective biserial algebras, Trans. Amer. Math. Soc. 330 (1992), 165-189 MR 93b:16022

[E1] K. ERdmann, Blocks of tame representation type and related algebras, Lecture Notes in Mathematics, 1428 (Springer 1990) MR 91c:20016

[E2] K. ERdmann, On Auslander-Reiten components for group algebras, J. Pure Appl. Algebra 104 (1995), 149-160 MR 97g:16026

[H] M. Hoshino, Modules without self-extensions and Nakayama's conjecture, Arch. Math. 43 (1984), 493-500

[K1] O. Kerner, Tilting wild algebras, Journal London Math. Soc. 39 (1989), 29-47 MR 90d:16025

[K2] O. Kerner, Stable components of wild tilted algebras, J. Algebra 142 (1991), 37-57 MR 92k:16021

[K3] O. Kerner, Representations of wild quivers, in Proc. of the Workshop on Representation theory of Algebras and related topics, UNAM, Mexico, 1994 CMS Conf. Proc. 19 (1996), 65-107 MR 97c: 16028

[KL] O. Kerner, F. Lukas, Regular modules over wild hereditary algebras, Proc. ICRA V, CMS Conf. Proc. 11 (1991), 191-208 MR 93c:16017 
[P] Z. Pogorzaly, On the stable Grothendieck groups, in Proc. ICRA VI, CMS Conf. Proc. 14 (1993), 393-406 MR 94f:16018

[R1] C.M. Ringel, Finite-dimensional hereditary algebras of wild representation type, Math. Z. 161 (1978), 235-255 MR 80c:16017

[R2] C.M. Ringel, Tame algebras and integral quadratic forms, Lecture Notes in Math. 1099 (Springer, 1984) MR 87f:16027

[W] P. WebB, The Auslander-Reiten quiver of a finite group, Math. Z. 179 (1982), 97-121 MR 83b:16029

Mathematical Institute, University of Oxford, 24-29 St. Giles, Oxford OX1 3LB, UNITED KINGDOM

E-mail address: erdmann@maths.ox.ac.uk

Mathematisches Institut, Heinrich-Heine-Universität, D-40225 Düsseldorf, Germany

E-mail address: kerner@cs.uni-duesseldorf.de 